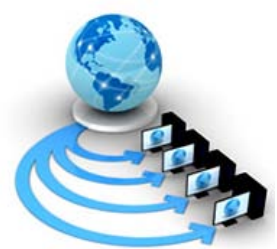

Volume 8, No. 7, July - August 2017

\title{
AN IMPROVEMENT OF PRIVACY PRESERVING USING BLOCK-TINY ENCRYPTION ALGORITHM: CLOUD APPROACH
}

\author{
Himanshu Kumar \\ School of information \& technology (SoICT) \\ Gautam Buddha University \\ Greater Noida (U.P)
}

\author{
Dr Anurag Singh Baghel \\ School of information \& technology (SoICT) \\ Gautam Buddha University \\ Greater Noida (U.P)
}

\begin{abstract}
In this paper we have a tendency to evaluate the economical, scalable, and sensible technique for privacy-preserving K-NN search. The approach allows the wide utilization of k-nearest neighbours search in confidential situations when none of the parties reveal their info whereas they'll still hand and glove notice the closest matches. To progress Block-Tiny Encryption Algorithm(TEA-AES) privacy conserving model for conserving the privacy of the patient's information in an exceedingly cloud aided system because the sensitive info is required to be maintained confidential and may not be discovered to public users apart from the physicians.
\end{abstract}

Keywords: Cloud approach, privacy preserving, TEA, KNN, MATLAB 2014a.

\section{I.INTRODUCTION}

The privacy preserving for the cloud assisted system is analyzed and the advantages of the protocol are determined. Privacy protection is an important aspect in the medical systems as their high risk of sensitive individual data being exposed to the public in an unauthorized way. The personal health information is collected from the patients with attributes such as heart beat rate, blood pressure, etc. during the medical treatment in terms of both text and images. An efficient privacy preserving fully homomorphism data aggregation is proposed to support both addition and multiplication operations. The dynamic medical data mining and the image feature extraction are the only processes that require privacy preserving data aggregation [3]. The privacy in data aggregation is achieved in this scheme by a tradeoff between the functionality and the optimized efficiency. Protection considerations arise when confidential information is outsourced to the cloud. By utilizing cryptography, the cloud server (i.e. its administrator) is prevented learning content in the outsourced databases. However, will we tend to additionally stop a neighborhood administrator from taking in the database content. Also, how might we keep away from situations, for example, workers utilizing cloud applications might learn quite it's required to playing their various duties? As an illustration, a company might want to specify rules limiting request-per-day for call center staff to one hundred customer contacts. Such limitations stop download of the entire (client) information. we present in this paper is a system design that enables comfortable and versatile restriction writing. Also, in doing as such, neighborhood directors and also cloud executives don't seem to be able to amendment the access rules when an application is launched. The paradigm shift involves/results within the loss of management over information also as new security and privacy problems [6]. Consequently, caution is suggested once deploying and utilizing Cloud computing in enterprises. After all, "the primary huge issue in information security in Europe arose at the end of the 1960's, once a Swedish organization chosen to have its information handling done by an administration agency in Germany and the information insurance legislations in each countries weren't alike. With Cloud Computing rapidly accomplishment approval, it is significant to focus the subsequent risks. As security and privacy problems square measure most vital, they must be addressed before Cloud Computing sets up a very important market share. In our work we proposed a new emerging concept namely KNN and TEA approach for data privacy preserving in cloud system [8, 9].

\section{A. KNN in a privacy preserving}

K-Nearest Neighbor privacy protective model for protective the protection of the patients in a cloud power-assisted framework because the sensitive data is required to be maintained classified and may not be unconcealed to public users aside from the doctor. Hence the privacy is modified by using k-nearest neighbor to develop K-Nearest Neighbor model in such a way that security of the medical data is improved. Instead of using a threshold value for the computed correlation function, the encrypted template $(\mathrm{T})$ and the encrypted medical data $(\mathrm{P})$ are processed to two non-colluding cloud service providers. The physician medical templates are encrypted and are outsourced to a cloud service provider while the secret key is stored in another cloud service provider.

\section{B. TEA technique}

Tiny Encryption Algorithm could be a Fiestal cipher that is utilizing numerous iterations instead of sophisticated programming. one-bit modification within the plain text will conjure to thirty-two bits modification within the Cipher Text. Tiny Encryption performs terribly with efficiency on trendy computers. the straightforward implementation of TEA has created it highly regarded. Tiny Encryption was executed using reduced key size of sixty-four bits rather than 128 bit [10].

\section{II.SYSTEM MODEL}

The (k-NN) technique, owing to its explainable nature, could be an easy and extremely intuitively appealing methodology to handle classification issues. However, selecting associate degree applicable distance operate for k-NN will be difficult associate degreed an inferior selection will create the classifier extremely liable to noise within the information. The great optimal of $\mathrm{k}$ depends upon the data; usually, greater values of $\mathrm{k}$ decrease the consequence of noise on the sorting, but produce boundaries between categories less distinct. a decent $\mathrm{k}$ may be elect by varied heuristic techniques. 
In binary classification difficulties, it is cooperative to select $\mathrm{k}$ to be an odd no. as this escapes tied votes. The K-NN algorithmic program is the easiest of all machine learning calculation: Associate in object is confidential by a larger part vote of its neighbors, with the item being allotted to the category most typical amongst its $\mathrm{k}$ nearest neighbors [1]. Normally, Euclidean distance is reused because the distance metric; although this is often appropriate to constant factors. In cases like text classification, another metric like the overlap metric or playing distance, as an example, is utilized.

K-Nearest Neighbor could be a modest procedure that provides all getable cases and categorizes new cases supported a correspondence live (e.g., distance functions). K-Nearest Neighbor has been utilized in applied mathematics estimation and pattern recognition already within the starting of 1970's as a non-parametric procedure.

$\mathrm{K}$ nearest neighbor formula is incredibly easy. It mechanism supported smallest distance from the question example to the training examples to control the K-nearest neighbors. The data for KNN algorithm consist of several attribute names that will be accustomed classify. the info of KNN will be any estimation scale from nominal, to quantitative scale [1,2].

The KNN algorithm is shown in the following form:

Input: $\mathrm{A}$, the arrangement of $\mathrm{k}$ preparing items, and test items $\mathrm{z}=\left(\mathrm{m}^{\prime}, \mathrm{n}^{\prime}\right)$.

Process: Compute $a\left(m^{\prime}, m\right)$, the separation $b / w ~ z$ and each item, $(\mathrm{m}, \mathrm{n}) \in \mathrm{A}$. Select $\mathrm{Az} \subseteq \mathrm{A}$, the arrangement of k nearest preparing items to $\mathrm{z}$.

Outcome: $\mathrm{n}^{\prime}=\operatorname{argmaxp} \sum(\mathrm{mi}, \mathrm{ni}) \subseteq \mathrm{Az} \mathrm{I}(\mathrm{p}=\mathrm{ni})$

- $\quad$ is a class label

- $\quad$ ni is the class label for the ith closest neighbors.

- $\{\mathrm{I}\}$ is AN indicator operate that returns the worth one if its argument is not false and zero generally.

In this system, KNN algorithm is used the suitable result by mixing the Euclidean distance among the various kinds of distance metric. The Euclidean distance is as shown in below:

$$
a_{i j}=\sqrt{\left(m_{i 1}-m_{j 1}\right)^{2}+\left(m_{i 2}-m_{j 2}\right)^{2}+\cdots+\left(m_{i p}-m_{j p}\right)^{2}}
$$

Where

aij $=$ the distance between the training objects and test object

$\mathrm{mi} \quad=$ input data for test object

$\mathrm{mj}$ = data for training objects stored in the database

\section{III.PROPOSED METHOD}

Block based Tiny encryption algorithm works as a Feistel system (a symmetric slab code) that usages a mixture of bit unstable, XOR, and enhance processes to generate the essential dispersal and misperception of data.

It fixes these processes on 32 bit arguments slightly than single bytes, an identical significant optimization that the authors avoid "progressive the authority of a processor." It customs a 128 bit (4 word) key, involvement in its separate word mechanisms in an actual key agenda [4,5].

The innovative operation works on 64 bits (two words) of facts at a time, though options (such as Block TEA) permit arbitrary-sized blocks.

\section{A. AES-128}

We confine to depiction of a characteristic round of advance encryption algorithm. Every round includes of four sub-processes [7]. The 1st round procedure is represented below: -Byte Substitution (Sub Bytes)The 16 input bytes are replaced in observing up a secure table (S-box) assumed in strategy. The consequence is in a matrix of 4 rows and 4 columns.

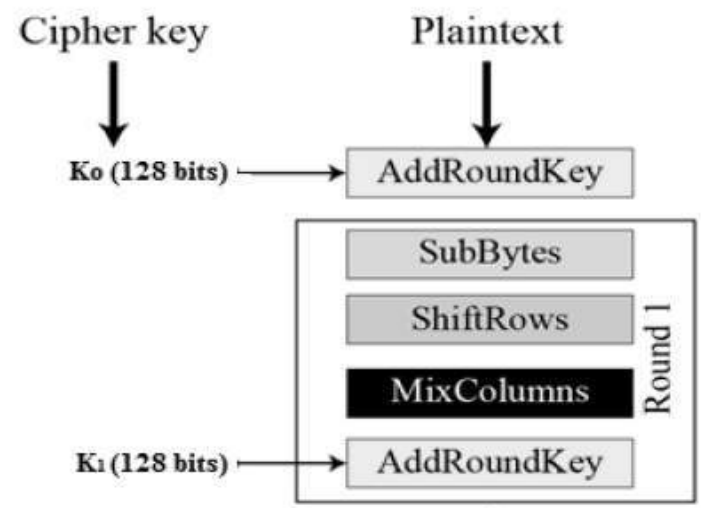

Fig. 1: Operation process of AES-128

\section{B. Shift rows}

All of the 4 rows of the matrix are removed to the left-hand. Some accesses that 'fall off' are re-inserted on the correct crosswise of row. Shift is approved out as surveys -

- $\quad 1$ st row is not removed.

- 2nd row is removed 1 (byte) location to the left.

- $\quad 3 r d$ row is removed 2 locations to the left.

- 4 th row is removed 3 positions to the left.

- The consequence is a novel matrix containing of the similar 16 bytes but removed w.r.t each other.

\section{Mix Columns}

Every column of 4 bytes is currently altered using a singular exact purpose. This purpose taking as input the four bytes of one column and productions four entirely new bytes, which change the unique column. The consequence is alternative novel matrix containing of 16 novel bytes. It must be wellknown that this stage is not achieved in the previous round [8].

\section{Add round key}

The 16 bytes of the matrix are presently dignified as 128 bits and are XORed to the 128 bits of the round key [9]. In case this is the most recent round formerly the productivity is the cipher text. Then, the subsequent 128 bits are construed as 16 bytes and we instigate additional comparable round.

\section{IV.RESULT}

This work presents the hybrid cryptography of the Tiny Encryption and AES-128 Set of rules. In this investigation we reviewed the best collective approaches in the cryptography of a slab cipher system. The resultant of Public-Key Processes is symmetric, that is to approximately use to encode the text or given text by user is different from the key used to decrypt the 
message. The encryption key, identified as the Public key which used to encode a communication, but the message can only be deciphered through the information that has the decryption key, recognized as the private key.

This type of encryption has a quantity of advantages over usual symmetric Ciphers. It means that the recipient can create their public key approximately available- someone deficient to send them a communication usage the procedure and the receiver's public key to do so. A viewer may have both the procedure and the public key, but will still not be capable to decode the text. Individual the receiver, with the private key can decrypt the message.

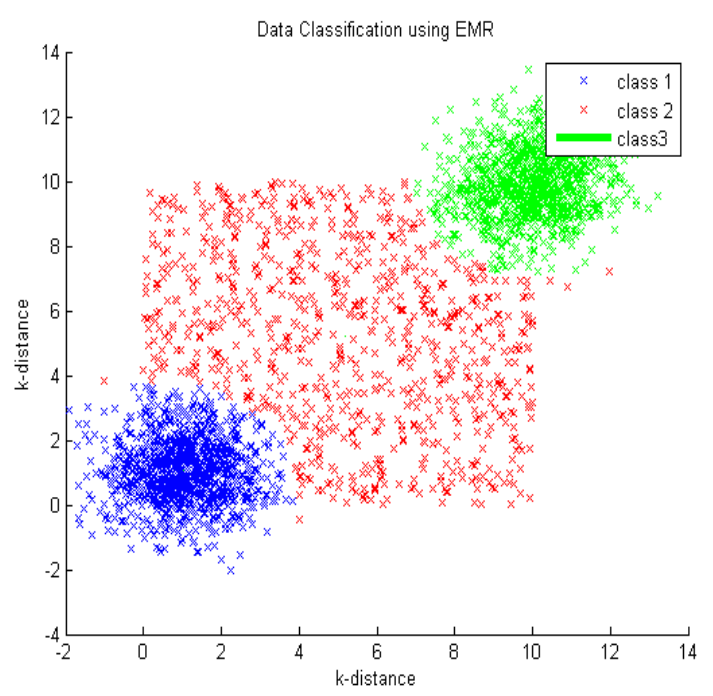

Figure 2: Data classification using EMR data

Figure 2 shows the classification of EMR data in three-part class 1 , class 2 and class 3 . It also measured the k-distance between one clusters to other cluster.

A circumstance is confidential through a large amount votes of its neighbors, with the case being allocated to the class almost mutual between its KNN measured by a separation work. If $\mathrm{K}$ $=1$, then the case is basically allocated to the class of its closest neighbor. Distance calculated by below operate.

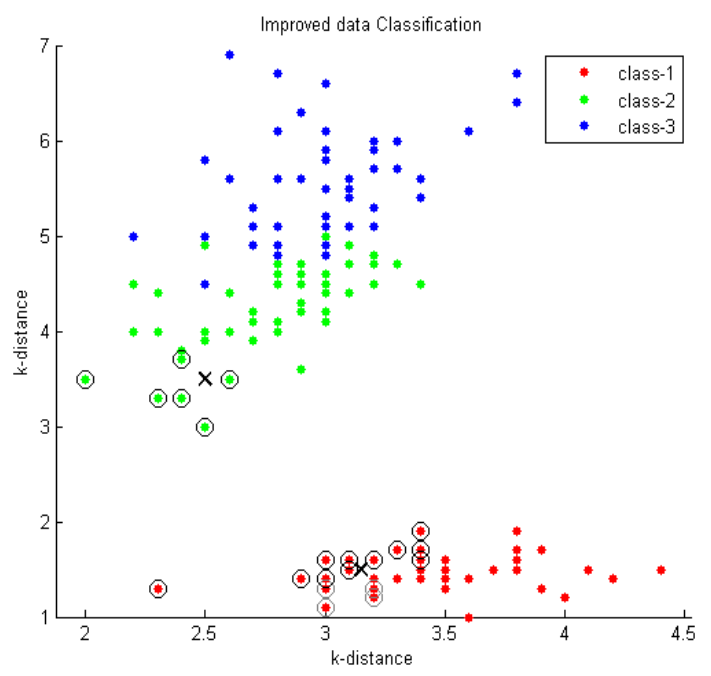

Figure 3: Data classification using KNN for EMR data

As above figure we can see that data classification is improved as compare to figure 3
Above figure shows the EMR taken more memory space as compare to proposed method.

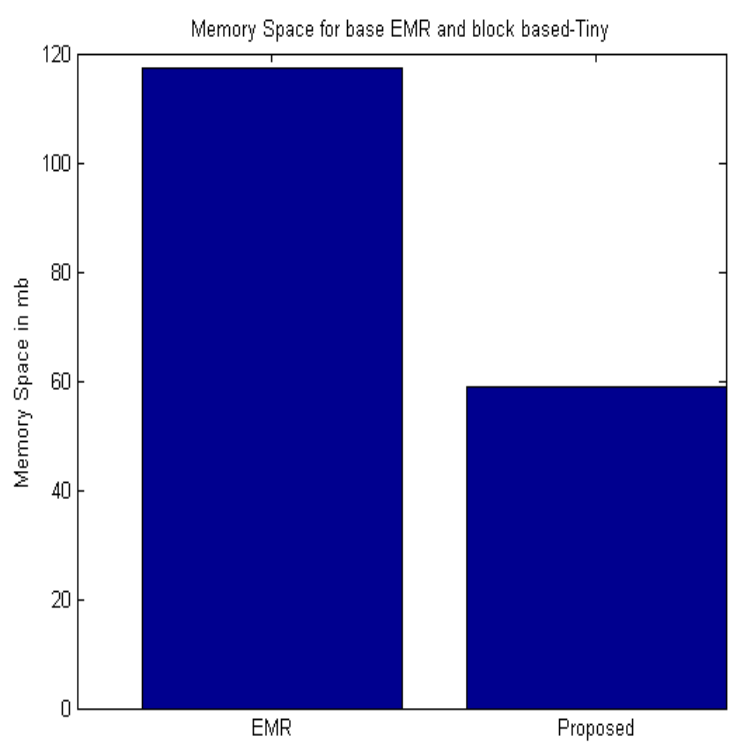

Figure 4: Memory space for base EMR and block based Tiny

The PDF is utilized to determine the chance of the variable quantity falling among a selected range of values, as opposed to taking on any one value. This prospect is given by the fundamental of this current variable's PDF over that range- it is given by the region under the density operate however on top of the horizontal axis and between the bottom and greatest values noteworthy estimations of the range. The prospect density operate is non negative everyplace, and its integral over the whole space is up to 1 .
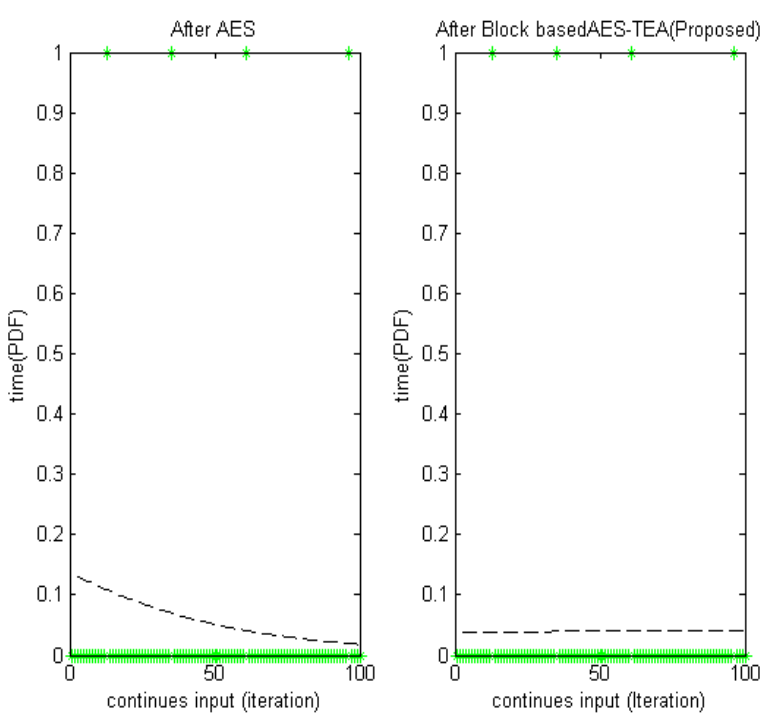

Figure 5: EMR and block based Tiny

\section{V.CONCLUSION}

Our proposed approach will define an emerging scheme in which two methods, AES and block based tiny which offers a robust support for its safety and the method to protected data or message with verification and signature verification in our hybrid method which goes to modify the innovation of the records files into encrypted form using Tiny-AES-128 encryption procedure that variations it into an illegible cipher text and plaintext is cryptography using the processes from 
mixed (orthogonal) arithmetical collections and an enormous amount of circles to attain security with easiness. At two, sixty-four (64) Feistel rounds, an entire number of rounds are used in the TEA-AES-128 encryption process with smallest time next encoded, the encoded files is embedding in a random text by using the idea of cryptography and formerly this text file directed via user and Processing time for block cipher, response time for senders, minimizing space consumption of s-box simulate in MATLAB.

\section{VI.REFERENCES}

[1] A. Andoni and P. Indyk. Near-optimal hashing algorithms for approximate nearest neighbor in high dimensions. In FOCS, 2006.J. Clerk Maxwell, A Treatise on Electricity and Magnetism, 3rd ed., vol. 2. Oxford: Clarendon, 1892, pp.68-73.

[2] M. Bellare, V. Tung Hoang, Sriram K., and P. Rogaway. Efficient garbling from a fixed-key block cipher. In S\&P. IEEE, 2013.K. Elissa, “Title of paper if known,” unpublished.

[3] J. Boyar and R. Peralta. Concrete multiplicative complexity of symmetric functions. In MFCS. Springer, 2006.
[4] Brenner, perl, and Smith. hcrypt Secure Function Evaluation (SFE) project. https://hcrypt.com/sfe/.

[5] H. Carter, C. Lever, and P. Traynor. Whitewash: Outsourcing garbled circuit generation for mobile devices. In ACSAC. ACM, 2014.

[6] H. Carter, B. Mood, P. Traynor, and K. Butler. Secure outsourced garbled circuit evaluation for mobile phones. In USENIX Security. USENIX, 2013.

[7] D. Demmler, T. Schneider, and M. Zohner. Ad-hoc secure twoparty computation on mobile devices using hardware tokens. In USENIX Security. USENIX, 2014.

[8] C. Gentry. Fully homomorphic encryption using ideal lattices. In STOC, 2009.

[9] A. Bessani, M. Correia, B. Quaresma, F. Andr'e, and P. Sousa, "Depsky: Dependable and secure storage in a cloud-of-clouds," in Proceedings of the Sixth Conference on Computer Systems, 2011, pp. 31-46

[10] E. Stefanov, M. van Dijk, A. Juels, and A. Oprea, "Iris: A scalable cloud file system with efficient integrity checks," in Proceedings of the $28^{\text {th }}$ Annual Computer Security Applications $\begin{array}{lll}\text { Conference, 2012, } & \text { 229-238 }\end{array}$ 